\title{
Adeno-associated virus capsid antigen presentation is dependent on endosomal escape
}

\author{
Chengwen Li, ${ }^{1,2}$ Yi He, ${ }^{1,3}$ Sarah Nicolson, ${ }^{1,4}$ Matt Hirsch, ${ }^{1}$ Marc S. Weinberg, ${ }^{1}$ \\ Ping Zhang, ${ }^{1}$ Tal Kafri, ${ }^{1,5}$ and R. Jude Samulski ${ }^{1,4}$
}

\begin{abstract}
${ }^{1}$ Gene Therapy Center and 2 Department of Pediatrics, University of North Carolina at Chapel Hill, Chapel Hill, North Carolina, USA.
${ }^{3}$ Hematopoietic Stem Cell Transplantation Center, Institute of Hematology, Chinese Academy of Medical Sciences, Tianjin, China.

${ }^{4}$ Department of Pharmacology and ${ }^{5}$ Department of Microbiology and Immunology, University of North Carolina at Chapel Hill, Chapel Hill, North Carolina, USA.
\end{abstract}

\begin{abstract}
Adeno-associated virus (AAV) vectors are attractive for gene delivery-based therapeutics, but data from recent clinical trials have indicated that AAV capsids induce a cytotoxic T lymphocyte (CTL) response that eliminates transduced cells. In this study, we used traditional pharmacological agents and AAV mutants to elucidate the pathway of capsid cross-presentation in AAV-permissive cells. Endosomal acidification inhibitors blocked AAV2 antigen presentation by over $90 \%$, while proteasome inhibitors completely abrogated antigen presentation. Using mutant viruses that are defective for nuclear entry, we observed a $90 \%$ decrease in capsid antigen presentation. Different antigen presentation efficiencies were achieved by selectively mutating virion nuclear localization signals. Low antigen presentation was demonstrated with basic region 1 (BR1) mutants, despite relatively high transduction efficiency, whereas there was no difference in antigen presentation between BR2 and BR3 mutants defective for transduction, as compared with wild-type AAV2. These results suggest that effective AAV2 capsid antigen presentation is dependent on AAV virion escape from the endosome/lysosome for antigen degradation by proteasomes, but is independent of nuclear uncoating. These results should facilitate the design of effective strategies to evade capsid-specific CTL-mediated elimination of AAV-transduced target cells in future clinical trials.
\end{abstract}

\section{Introduction}

Adeno-associated virus (AAV) is a single-stranded DNA virus with a genome composed of the rep and capsid genes flanked by 2 inverted terminal repeats. AAV vectors have been successfully used in several clinical trials in patients with Leber congenital amaurosis and hemophilia B (1-6). Gene delivery using AAV vectors is attractive due to their ability to transduce dividing and nondividing cells, their ease of production, their long-term transgenic expression, and their lack of pathogenicity. AAV vectors are constructed by substituting the rep and capsid genes with therapeutic ones. Since there are no viral genes in AAV vectors, it has been postulated that cellular immune responses to AAV may be low. However, recent data from a clinical trial suggested that AAV capsid-specific cytotoxic T lymphocytes (CTLs) may eliminate AAV-transduced target cells. In 1 patient with hemophilia B, therapeutic protein levels were obtained 4 weeks after liver transduction of an AAV serotype 2 (AAV2) vector encoding coagulation factor IX (F9). Unexpectedly, however, the F9 levels remained high for only 2 weeks, and then declined back to basal levels, with concomitant elevation of liver transaminases, indicating liver damage caused by a CTL immune response. Further experiments have suggested that a capsid-specific CTL response contributed to this outcome $(5,6)$.

Conflict of interest: The authors have declared that no conflict of interest exists.

Note regarding evaluation of this manuscript: Manuscripts authored by scientists associated with Duke University, The University of North Carolina at Chapel Hill, Duke-NUS, and the Sanford-Burnham Medical Research Institute are handled not by members of the editorial board but rather by the science editors, who consult with selected external editors and reviewers.

Citation for this article: J Clin Invest. 2013;123(3):1390-1401. doi:10.1172/JCI66611.
Indeed, in mouse models, using an adenovirus vector to deliver the AAV capsid, direct intramuscular delivery of AAV, or application of AAV vector-pulsed dendritic cells (7-9) can elicit a CTL response against the AAV capsid. These results indicate that AAV capsid antigen can be presented via both classical antigen presentation and cross-presentation pathways. In humans and primates, it has been demonstrated that a capsid-specific CTL response is induced from natural AAV2 infection based on a sensitive IFN- $\gamma$ ELISPOT analysis (10).

Antigen cross-presentation from exogenous protein has been intensively studied in professional APCs. Two distinct working models for the cross-presentation of exogenous antigens on MHC class I molecules have been proposed (11). The first pathway (cytosolic pathway) utilizes the classical endogenous antigen-processing machinery to generate antigenic peptides. After exogenous protein is taken up by endocytosis, antigen makes its way into the cytosol where it is degraded by the proteasome before being translocated into the ER by the transporter associated with antigen presentation (TAP). In the ER, the peptide antigen is loaded onto nascent MHC I molecules to form antigen-MHC I complexes which are then presented on the cell surface to activate $\mathrm{CD}^{+} \mathrm{T}$ cells (12). In the second pathway (vacuolar or endosomal pathway), endocytosed antigen is processed independently of the proteasome and the TAP. The protein is degraded by proteases within the endosomal-lysosomal system and loaded onto recycled MHC I molecules, similar to the MHC class II antigen presentation pathway $(13,14)$. Although AAV-transduced hepatocytes are killed by capsid-specific CTLs with identical MHC class I alleles, and proteasome inhibition protects target cell killing by these CTLs $(5,15)$, no detailed stud- 
A $\operatorname{CD} 69$
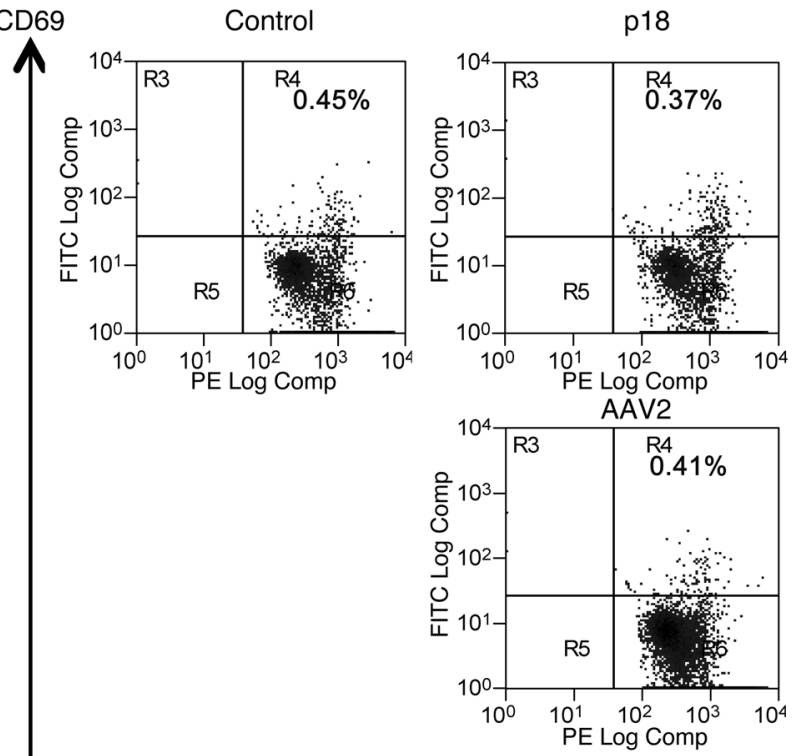
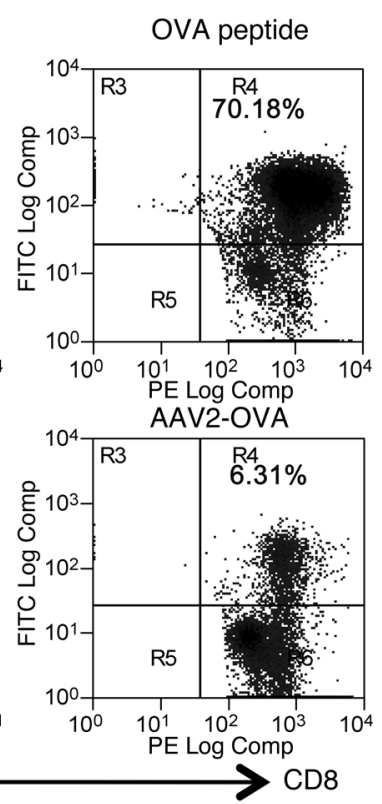

B

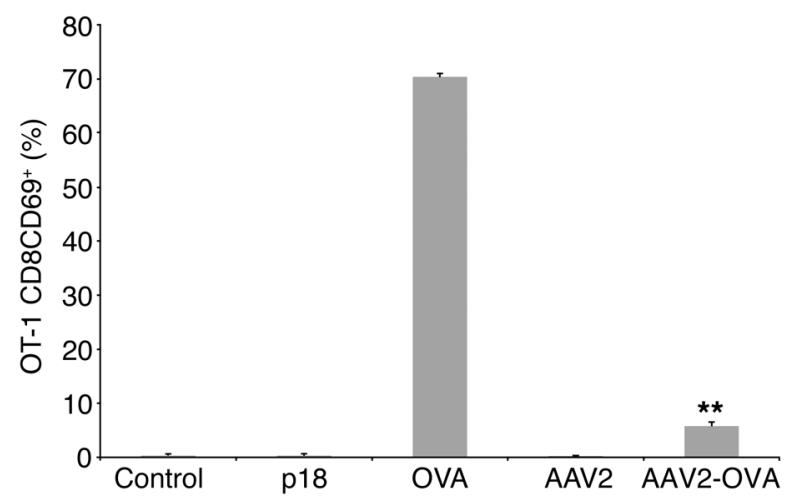

ies have been carried out to determine the mechanism of AAV capsid antigen cross-presentation in AAV2-transduced cells.

$\mathrm{AAV}$ transduction involves several steps, including AAV binding on the target cell surface, receptor-mediated endocytosis into an endosome and lysosome, perinuclear accumulation, entrance into and uncoating within the nucleus, and second-strand synthesis before transgenic expression (16-18). AAV2 infection requires heparan sulfate proteoglycan (HSPG) as a primary receptor, together with coreceptors which include FGF receptors, integrin receptors, laminin receptors, or HGF receptors for optimal attachment (19-24). Following attachment to cell surface receptors, AAV2 internalization occurs via a receptor-mediated endocytotic mechanism. The process of endocytosis is clathrin and dynamin dependent $(25,26)$. Endosomal acidification is necessary for effective AAV2 infection. In the endocytic system, AAV2 virion is trafficked to lysosomes through early endosomes, late endosomes, and recycling endosomes $(17,26)$. In the acidic environment, the AAV2 virion undergoes a conformational change to expose several domains in VP1 and/or VP2 N-termini, which are buried inside the capsid of the intact virion. Several domains assist the AAV2 virion to escape from the endosomal-lysosomal system and travel to the nucleus (27-31). These include a phospholipase A2 (PLA2) motif and 3 basic regions (BR1, 2, and 3), all of which putatively control endosomal escape, nuclear localization, and nuclear import via the nuclear pore complex, respectively (27-31). AAV2 particles have also been found to accumulate in the Golgi complex (32). It is generally accepted that AAV2 virion uncoating occurs in the nucleus where gene expression is then induced $(18,31)$. However, it remains unknown which organelles are responsible for generation of AAV2 capsid antigens. In this study, we applied classical pharmacological inhibitors to block different pathways of antigen processing and presentation in AAV2-transduced target cells and demonstrated that antigen presentation by AAV2 capsids is proteasome dependent. More importantly, using AAV2 mutants, which result in differential organelle trapping of virions after infection, we demonstrated that effective AAV2 capsid antigen presentation requires escape from the endosomal-lysosomal system and encapsidation of AAV2 genomes, independent of intact virion uncoating in the nucleus.

\section{Results}

Inbibition of endosomal acidification decreases $A A V$ capsid antigen presentation. To investigate the mechanism of AAV capsid antigen presentation and its relationship with AAV virion uncoating and degradation, we established a novel system by introducing a strong immune domain into the AAV2 capsid for detection of antigen-specific CTL activation in AAV2-permissive cells. OVA SIINFEKL peptide was used to replace the HI loop of AAV2 and maintained 

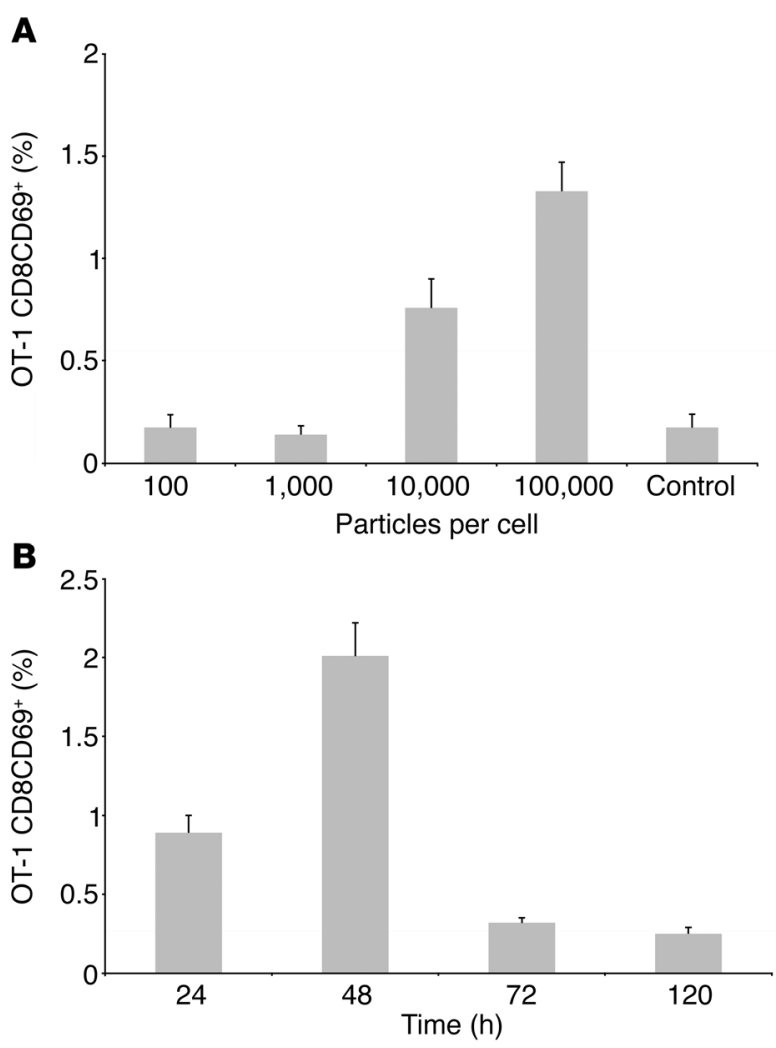

a similar capacity to package the AAV2 virion and transduce cells, as compared with wild-type AAV2 (33). We established HepG2/ $\mathrm{H}-2 \mathrm{~Kb}$ and $293 / \mathrm{H}-2 \mathrm{~Kb}$ cell lines by infection of lentivirus vector lenti/H-2Kb, which encodes the $\mathrm{H}-2 \mathrm{~Kb}$ transgene (Supplemental Figure 1; supplemental material available online with this article; doi:10.1172/JCI66611DS1). To address whether the OVA SIINFEKL peptide was processed and presented on the surface of $\mathrm{HepG} 2 / \mathrm{H}-2 \mathrm{~Kb}$ with $\mathrm{H}-2 \mathrm{~Kb}$ molecules after AAV2-OVA infection, we transduced HepG2/H-2Kb cells by AAV2-OVA/AAT or AAV2/AAT viruses. Additionally, the OVA-derived SIINFEKL peptide and p18 from an alternative ORF of human coagulation factor IX cDNA were used to pulse HepG2/H-2Kb cells (34). Twenty-four hours later, HepG $2 / \mathrm{H}-2 \mathrm{~Kb}$ cells were fixed and washed thoroughly, and OT-1 spleen cells were added to HepG2/H-2Kb cells overnight. The activation of OT-1 spleen cells was detected by flow cytometry for expression of CD8 and the early activation marker CD69. As shown in Figure 1, the p18 peptide-pulsed cells did not activate OT-1 cells, but OVA SIINFEKL peptide treatment led to 70\% CD8/ CD69 double positivity. We observed no activation of OT-1 cells from AAV2/AAT transduction, similar to control and peptide $\mathrm{p} 18$ treatment $(0.3 \%, 0.36 \%$, and $0.37 \%$ of activated OT- 1 cells, respectively). In contrast, AAV2-OVA/AAT transduction induced $5.8 \%$ of activated OT-1 cells (Figure 1). This indicates that the SIINFEKL epitope from AAV2-OVA vector transduction can be processed and presented on the cell surface with $\mathrm{H}-2 \mathrm{~Kb}$ molecules and can activate CTLs. Previous studies have shown that acid neutralization in the endosomes inhibits AAV transduction (26), and we verified that such acid neutralization does not influence AAV entry (Supplemental Figure 2). To address whether inhibition of acidification in the endosomes affects antigen presentation by the AAV capsid after AAV transduction, we incubated HepG $2 / \mathrm{H}-2 \mathrm{~Kb}$ cells with

\section{Figure 2}

Capsid antigen presentation in AAV2-OVA-transduced HepG2/H-2Kb cells. (A) Dose response of antigen presentation. HepG2/H-2Kb cells $\left(2 \times 10^{5}\right.$ cells $)$ were seeded on a 12-well plate, then infected with AAV2-OVA/AAT virus at different doses. Forty-eight hours later, $1 \times 10^{6}$ spleen cells from OT-1 mice were added to cell culture overnight. The number of cells expressing CD8 and CD69 were detected by flow cytometry. (B) Time course of antigen expression. After HepG2/ $\mathrm{H}-2 \mathrm{~Kb}$ cells were plated, $1 \times 10^{5}$ particles of AAV2-OVA/AAT vector per cell were added to the culture medium. At different time points after AAV transduction, HepG2/H-2Kb cells were fixed with $1 \%$ paraformaldehyde in PBS and washed thoroughly. After incubation with OT-1 spleen cells, the activation of OT-1 spleen cells was detected by flow cytometry for expression of CD8 and CD69.

ammonium chloride or chloroquine for 1 hour before AAV2-OVA vector was added at $10^{5}$ per cell, since the efficiency of AAV capsid antigen presentation was dose dependent (Figure 2, A and B). After 24 hours of AAV transduction, the cells were fixed and washed. The spleen cells from OT-1 mice were cocultured with AAV2-OVAtransduced HepG2/H-2Kb cells overnight, and activated OT-1 spleen cells were detected by flow cytometry. The concentrations of ammonium chloride and chloroquine used in this experiment were not toxic to HepG2 cells (Figure 3A). Consistent with prior work, both ammonium chloride and chloroquine inhibited AAV2 transduction (Figure 3B) (26). Compared with antigen presentation from AAV2-OVA transduction without treatment, over $90 \%$ inhibition of antigen presentation was observed in the groups treated with ammonium chloride and chloroquine (Figure 3, C and D). In the endosome-lysosome system, protein is degraded by proteases, especially cathepsins. To analyze the participation of proteases in the processing of AAV capsid after transduction, we tested 2 different protease inhibitors: leupeptin $\mathrm{H}$ (an inhibitor of serine and cysteine proteases, including cathepsin B), and pepstatin A (an inhibitor of aspartate proteases, including cathepsins D and E) $(35,36)$. Incubation of HepG2/H-2Kb with AAV2OVA vector in the presence of leupeptin and pepstatin partially blocked OVA presentation, while no cell toxicity was observed (Figure 3, C and E), suggesting that OVA antigen processing from AAV2-OVA virion required the participation of some proteases. In disagreement with the results from Akache's study (37), we did not find that protease inhibitors had enhanced function in AAV transduction (Figure $3 \mathrm{~B}$ ), perhaps because different protease inhibitors or cellular targets were used (37).

$A A V$ capsid antigen presentation is dependent on proteasomal degradation. The proteasome is a multisubunit complex responsible for the complete degradation of many cellular proteins. In addition to its critical role in a variety of biological processes, the proteasome is also required for degradation of proteins to generate antigens for presentation to CTLs via the MHC class I pathway. To evaluate whether proteasomal degradation participates in AAV capsid antigen processing, we treated $\mathrm{HepG} 2 / \mathrm{H}-2 \mathrm{~Kb}$ cells with 2 proteasome inhibitors: MG132 and bortezomib. As with many previous studies (38), both proteasome inhibitors enhanced AAV2 transduction in HepG2 cells (Figure 3B). However, OVA antigen presentation by HepG $2 / \mathrm{H}-2 \mathrm{~Kb}$ cells incubated with AAV2-OVA vector in the presence of these inhibitors was completely diminished (Figure 3, $\mathrm{C}$ and $\mathrm{F}$ ). This result indicates that AAV2-OVA virions or derived peptides translocate to the cytosol of $\mathrm{HepG} 2 / \mathrm{H}-2 \mathrm{~Kb}$ cells and are degraded by the proteasomes. 
A

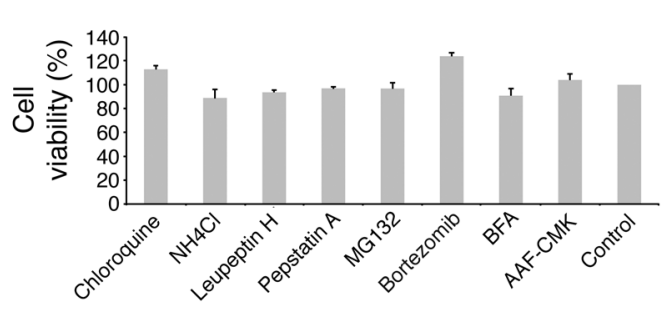

B
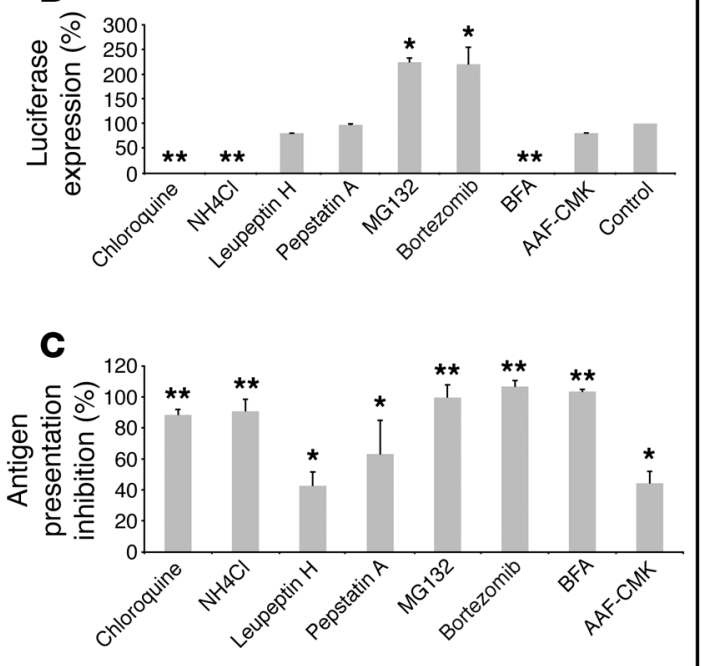

\& $\mathbf{D}$

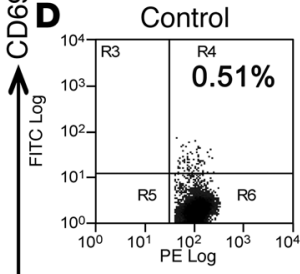

AAV2-OVA
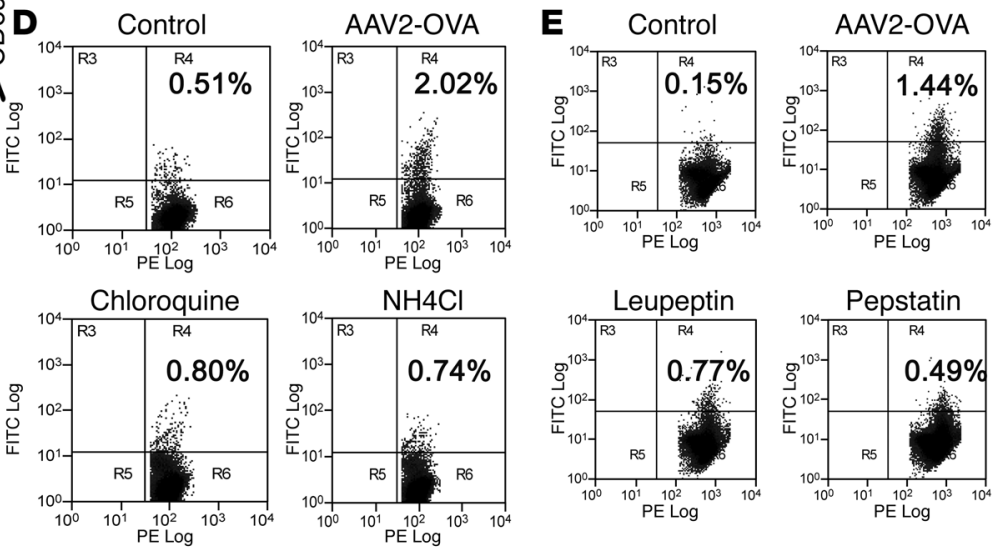
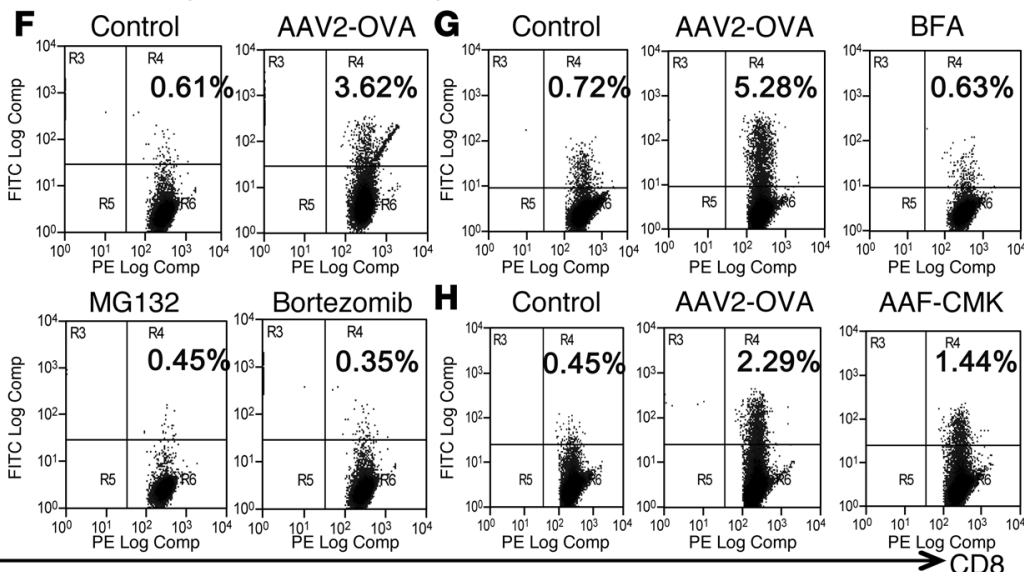

\section{Figure 3}

The effect of inhibitors on capsid antigen cross-presentation in HepG2/H-2Kb cells. (A) Toxicity of inhibitors on HepG2/H-2Kb cells. HepG2/H-2Kb cells $\left(2 \times 10^{5}\right.$ cells) were seeded on 12-well plates and incubated with different inhibitors. Cell viability was measured 24 hours later. (B) Effect of inhibitors on AAV2 transduction in HepG2/H-2Kb cells. HepG2/H-2Kb cells were incubated with inhibitors for 1 hour, then $1 \times 10^{4}$ particles per cell of AAV2-OVA/luc vector were added to the culture for 24 hours. Cells were harvested for luciferase analysis. ${ }^{*} P<0.05$ and ${ }^{* *} P<0.01$ when compared with AAV2/luc transduction without drug treatment. (C) Effect of inhibitors on antigen presentation. HepG2/H-2Kb cells were incubated with inhibitors for 1 hour and then transduced with $2 \times 10^{10}$ AAV2-OVA/AAT vector for 24 hours. Cells were then fixed with $1 \%$ paraformaldehyde and washed, and OT-1 spleen cells were added to HepG2/H-2Kb cell culture medium. Activation of OT-1 spleen cells was determined by flow cytometry detecting CD8 and CD69 expression. The inhibition of antigen presentation was calculated as: [1-(number of activated OT-1 cells in inhibitor group with AAV2-OVA infection minus background group without AAV2-OVA infection)/(number of activated OT-1 cells in the group with AAV2-OVA infection - background group)] $\times 100 \%$. ${ }^{\star} P<0.05$ and $P<0.01$ when compared with the AAV2-OVA group without treatment. Data represent the average of 4 individual experiments and standard deviations. (D-H) Representative data from 1 experiment for acidification inhibitor (D), protease inhibitor $(\mathbf{E})$, proteasome inhibitor $(\mathbf{F})$, Golgi transporter inhibitor $(\mathbf{G})$, and TPPII inhibitor $(\mathbf{H})$.

Tripeptidyl peptidase II is involved in AAV capsid antigen presentation. In addition to the proteasome, the cytosolic protease tripeptidyl peptidase II (TPPII) has been implicated in MHC class I antigen presentation. TPPII is the largest known multifunctional eukaryotic peptidase (39). In addition to its housekeeping function in intracellular protein degradation, TPPII also plays a role in several vital cellular processes including antigen processing (40). The chemical inhibitor of TPPII, AAF-MCK, led to approximately $50 \%$ blocking of antigen presentation by AAV2-OVA-transduced HepG2/ $\mathrm{H}-2 \mathrm{~Kb}$ cells, but without effect on cell toxicity or transduction efficiency (Figure 3, A-C, and $\mathrm{H}$ ).

AAV2 capsid antigen processing is sensitive to brefeldin A. Brefeldin A (BFA) inhibits the transport of proteins from the ER to the Golgi complex and blocks the secretion of newly synthesized proteins $(41,42)$. In this study, we used BFA to determine whether antigen processing and presentation from AAV2-OVA-infected cells was related to the transport of processed peptides through the Golgi complex to the HepG2/H-2Kb cell membrane. BFA significantly suppressed AAV2 transduction (Figure 3B), and incubation of HepG $2 / \mathrm{H}-2 \mathrm{~Kb}$ cells with BFA inhibited antigen presentation from AAV2-OVA transduction by nearly $100 \%$ (Figure 3, C and G). This strongly suggests that AAV2-OVA-derived peptides are transported to the cell surface through the Golgi complex after translocation to the ER.

Endosomal/lysosomal escape plays a role in AAV capsid antigen presentation. Major domains on the N termini of VP1 and VP2 in the capsid are necessary for AAV subcellular traveling. During infection (prior to endosomal/lysosomal escape), a conformational change promotes exposure of these motifs on the surface of the capsid (27-31). Several functional motifs have been identified: a PLA2 domain and putative nuclear localization signals (NLSs) BR1, BR2, and BR3. Phospholipase plays a role in disrupting the membrane of a vesicular compartment and allows for viral escape from the endosome/ 

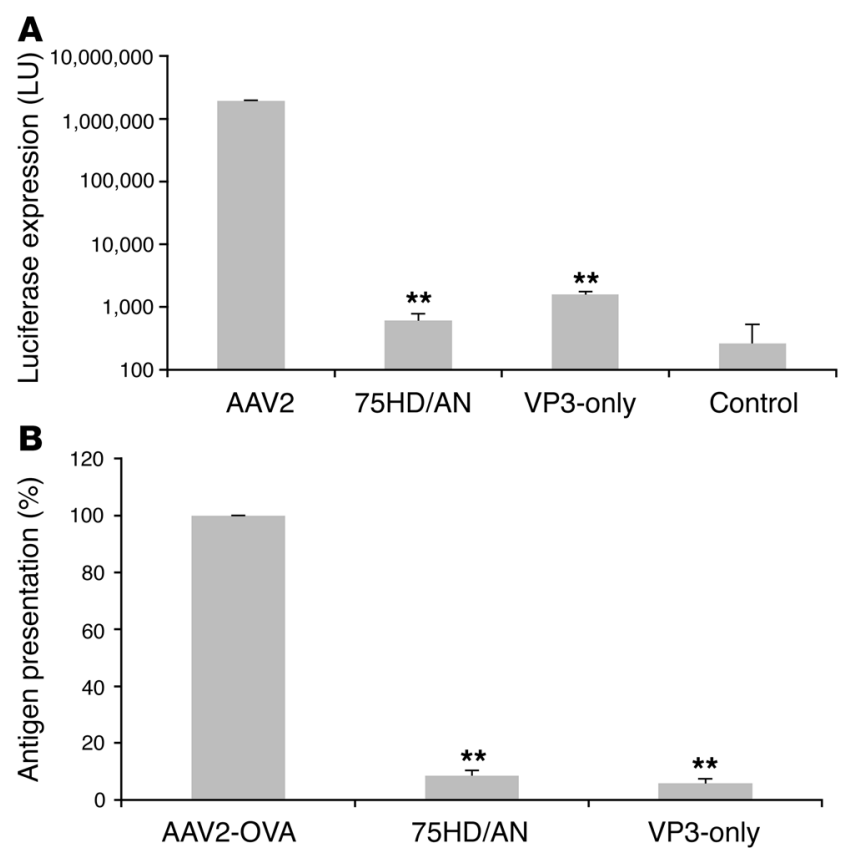

lysosome into the cytosol. It has been demonstrated that a mutant with a 2-residue substitution of PLA2 (AAV2/75HD/AN) is unaffected with regard to cell surface binding capacity or viral endocytosis, but has greatly attenuated PLA2 activity and compromised viral infectivity $(27,31)$. To further confirm the role of AAV endosomal/lysosomal escape in capsid antigen presentation, we incubated AAV2-OVA/75HD/AN vectors with HepG2/H-2Kb cells. Twenty-four hours later, the HepG2/H-2Kb cells were washed and then incubated with OT-1 spleen cells for another 24 hours, and activated OT-1 T cells were measured by flow cytometry. As shown in Figure 4, compared with the AAV2-OVA vector, a significant reduction in antigen presentation was observed with the AAV2-OVA/75HD/AN mutant vector (Figure 4B and Supplemental Figure 3). Similar to prior findings, the PLA2 mutant showed decreased AAV transduction (Figure 4A). Our previous study demonstrated that a VP3-only virus, which does not contain the PLA2 domain on its N-terminus, is also unable to enter the nucleus and fails to transduce AAV2-permissive cells. Similar to the AAV2-OVA/75HD/AN mutant, infection with AAV2-OVA VP3-only virus led to reduced antigen presentation by HepG2/ $\mathrm{H}-2 \mathrm{~Kb}$ cells (Figure 4 and Supplemental Figure 3 ).

Modification of $A A V$ capsid NLSs impacts antigen presentation. Three potential NLSs on the AAV capsid protein N-termini of VP1 and VP2 have been identified, namely, BR1 (only in VP1), BR2, and BR3

\section{Figure 5}

Effect of AAV NLSs on capsid antigen presentation. (A) Transgenic expression from transduction with 3 NLS mutants. HepG2 cells were transduced with $3 \mathrm{AAV}$ mutants (BR1, BR2, and BR3) at $1 \times 10^{4}$ particles per cell. Twenty-four hours later, AAV-based luciferase expression was measured. ${ }^{* \star} P<0.01$ when compared with AAV2. (B) Antigen presentation by transduced NLS mutants. HepG2/H-2Kb cells were transduced with NLS mutant vectors at $1 \times 10^{5}$ particles per cell for 24 hours. Cells were washed and incubated with $1 \times 10^{6}$ spleen cells of OT-1 mice overnight. Activation of CD8 T cells was detected by flow cytometry. ${ }^{* *} P<0.01$ when compared with AAV2-OVA. Data represent the average and standard deviations from 3 individual experiments.

\section{Figure 4}

Mutation of the PLA domain and VP3-only virus reduces AAV capsid antigen presentation. (A) Transgenic expression from an AAV2/75HD/ AN mutant and VP3-only viruses in HepG2 cells. HepG2 cells $\left(1 \times 10^{5}\right.$ cells) were seeded in 24-well plates, and $1 \times 10^{9}$ particles of AAV/ luc virus (AAV2, AAV2/75HD/AN, and VP3-only) were added to cell culture medium. After incubation for 24 hours, luciferase expression in AAV-transduced HepG2 cells was measured. ${ }^{* *} P<0.01$ when compared with AAV2. (B) Antigen presentation by transduced AAV2-OVA, $75 \mathrm{HD} / \mathrm{AN}$, and VP3-only virus. Particles $\left(2 \times 10^{10}\right.$ particles $)$ of AAV2OVA viruses (AAV2-OVA, AAV2-OVA/75HD/AN, and AAV2-OVA/VP3only) were incubated with $2 \times 10^{5} \mathrm{HepG} 2 / \mathrm{H}-2 \mathrm{~Kb}$ cells for 24 hours, after which unbound virus was washed out. Spleen cells $\left(1 \times 10^{6}\right.$ cells $)$ from OT-1 mice were added to HepG2/H-2Kb culture medium and incubated overnight. Reduction of antigen presentation by the PLA mutant and transduced VP3-only virus were calculated as described in Methods. ${ }^{* *} P<0.01$ when compared with AAV2-OVA. Data are based on the average and standard deviations from 3 individual experiments.

(in VP1 and VP2); these NLSs are comprised of hydrophilic, basic regions (also considered basic clusters) (29-31). We have demonstrated that mutation of these regions dramatically decreases transduction as a result of defective nuclear entry of the mutant virus (30). An imaging study of AAV2 trafficking has shown that the BR1 mutant localizes mainly to the Golgi complex, whereas BR2 and BR3 mutants display a diffused localization, distal from the nucleus after infection (30). To understand the relationship between AAV capsid antigen presentation and uncoated AAV capsid degradation localized within organelles, we integrated the OVA SIINFEKL peptide into NLS mutants by substitution of the HI loop. Similar to our prior result, compared with AAV2-OVA vector, the OVA integration mutants reduced the transduction efficiency of BR1, BR2, and BR3 mutants by 4-, 100-, and 2,000-fold (Figure 5A), respectively. Next, we infected $\mathrm{HepG} 2 / \mathrm{H}-2 \mathrm{~Kb}$ cells with these

A

B
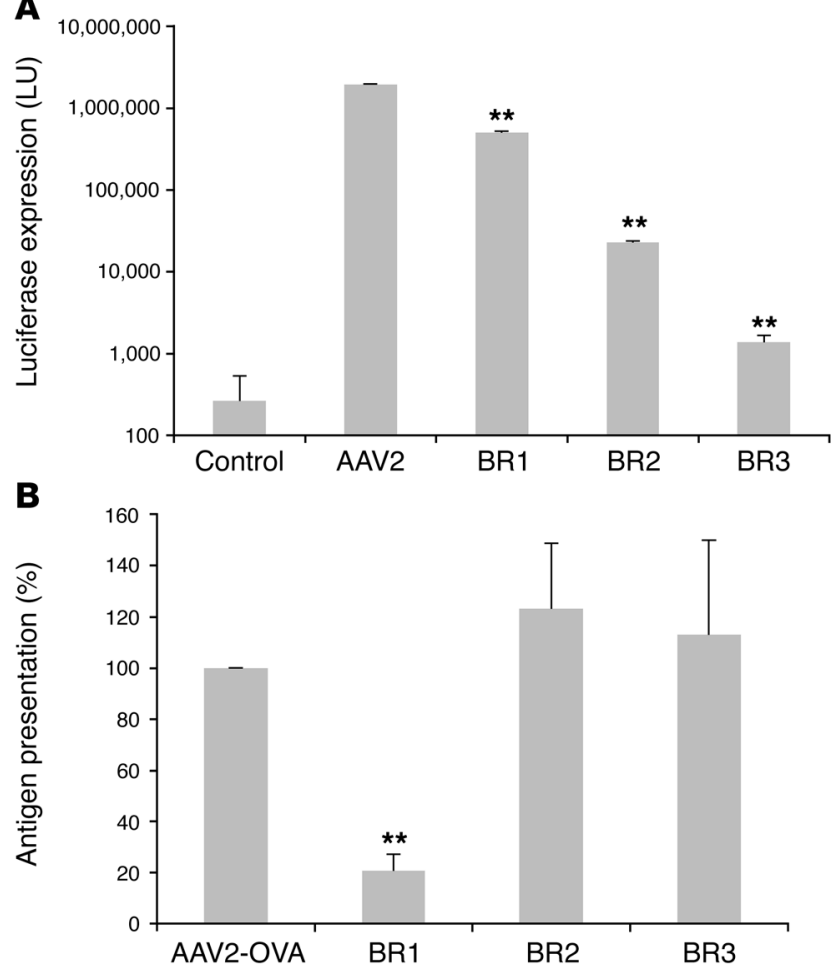


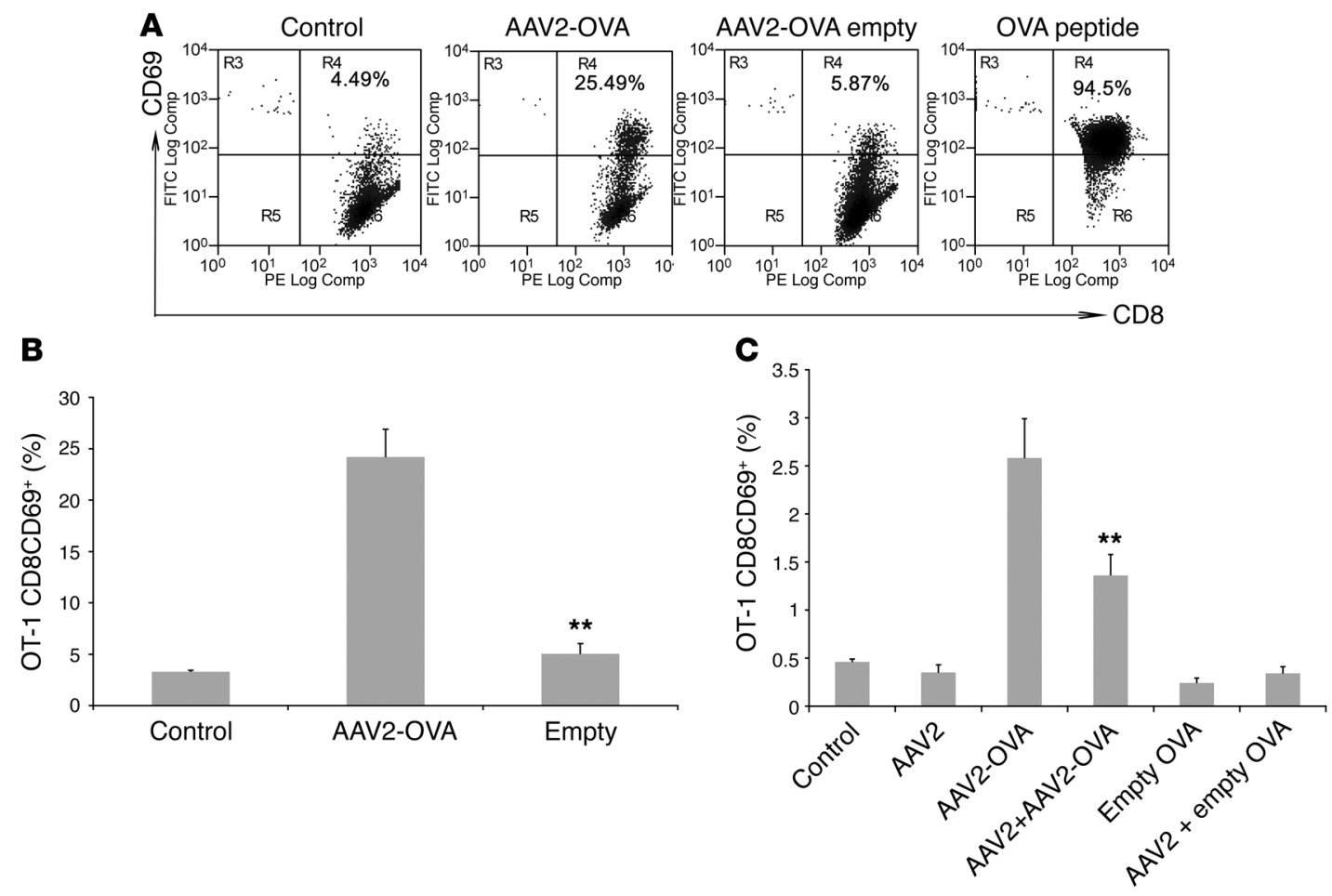

\section{Figure 6}

Capsid antigen cross-presentation from empty virus infection. HepG2/H-2Kb cells $\left(2 \times 10^{5}\right.$ cells $)$ were transduced with $1 \times 10^{5}$ particles per cell of empty virus for 24 hours. Cells were then washed and incubated with OT-1 spleen cells overnight. The activation of CD8 T cells was detected by flow cytometry. (A) Graphs are representative data sets from 1 experiment. (B) Decreased antigen presentation from empty virion transduction. ${ }^{* \star} P<0.01$ when compared with AAV2-OVA. (C) No effect of AAV2 full particles on antigen presentation by empty OVA capsids. HepG2/H-2Kb cells $\left(2 \times 10^{5}\right.$ cells $)$ were transduced with AAV2/AAT and AAV2-OVA or empty OVA virus at $5 \times 10^{4}$ particles per cell for each virus for 24 hours. Activation of OT- 1 spleen cells was detected after incubation of OT-1 cells with AAV-treated HepG2/H-2Kb cells. ${ }^{*} P<0.01$ when compared with AAV2-OVA. Data are based on the average and SD from 4 individual experiments.

mutants and examined antigen presentation on the cell surface. BR1 mutant infection led to significantly decreased antigen presentation. Interestingly, both BR2 and BR3 mutants induced antigen presentation similar to that induced by the AAV2-OVA vector (Figure 5 and Supplemental Figure 4). The result, combined with transduction efficiency, suggests that uncoating in the nucleus is not required for $A A V$ capsid antigen presentation after infection.

Effective $A A V$ capsid antigen presentation is dependent on the encapsidation of the $A A V$ genome. AAV vectors purified from $\mathrm{CsCl}$ have been applied in clinical trials, however this purification approach is not scalable. Recently, ion exchange chromatography has been studied to purify AAV vectors (43-46). Unlike the $\mathrm{CsCl}$ purification approach, the chromatographic method cannot currently separate genome-containing particles of AAV vectors (full particles) from empty virions because the virion surfaces are the same. The contamination of empty virions inhibits transduction from full particles of AAV vectors and potentially increases the virus capsid antigen load in transduced cells (46). To investigate the possibility of antigen presentation by empty particles, we made empty AAV vector by transfecting only 2 plasmids (pXX6-80 and pXR2OVA) instead of using 3 plasmids for AAV vector production. Virus titer was quantitated by Western dot-blot analysis with monoclonal antibody A20, which only recognizes intact AAV virion. The same particles of empty and full AAV2-OVA vectors were used to infect HepG $2 / \mathrm{H}-2 \mathrm{~Kb}$ cells to determine their antigen presentation capac- ity. Unexpectedly, despite the similarity of components (composition of VP1, VP2, VP3, PLA2, and NLS motifs) in empty and full particle virions of AAV vectors, significantly less antigen presentation by empty virions was induced than was the case with genomecontaining full particles (Figure 6). This result indicates that encapsidation of the AAV genome in AAV virions helps with AAV uncoating for proteasomal degradation. Generally in recombinant AAV preparations, most vector lots are a mixture of full and empty particles. To elucidate whether full particles have an effect on antigen presentation by empty capsids, we transduced HepG $2 / \mathrm{H}-2 \mathrm{~Kb}$ cells with the mixture of AAV2/AAT and empty OVA vectors and examined the activation of OT-1 spleen cells after incubation with the infected HepG2/H-2Kb cells. The incubation of AAV2/AAT vector with AAV2-OVA full particles inhibited AAV2-OVA capsid antigen presentation by 50\%-60\% (Figure 6C and Supplemental Figure 5). However, HepG2/H-2Kb cells transduced with AAV2/AAT and empty OVA vectors only induced $0.36 \%$ of the OT-1 CD8CD69activated cells, similar to the result seen in $\mathrm{HepG} 2 / \mathrm{H}-2 \mathrm{~Kb}$ cells treated with AAV2/AAT, empty OVA capsids, or control $(0.22 \%, 0.28 \%$, and $0.46 \%$, respectively) (Figure 6C and Supplemental Figure 5). This result suggests that wild-type AAV2 may not rescue AAV capsid antigen presentation by empty virions.

Proteasome-dependent degradation of AAV capsid in other cells. We demonstrate that AAV-transduced capsid antigen presentation is proteasomal degradation dependent in the human liver HepG2 
A

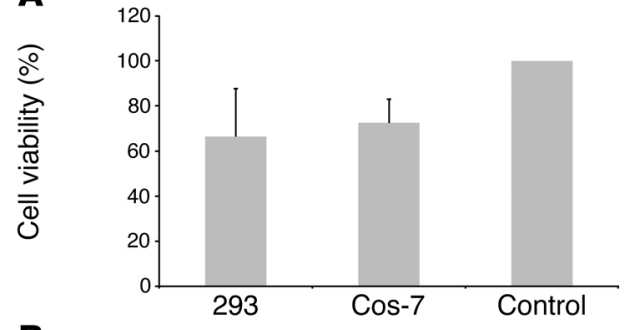

B

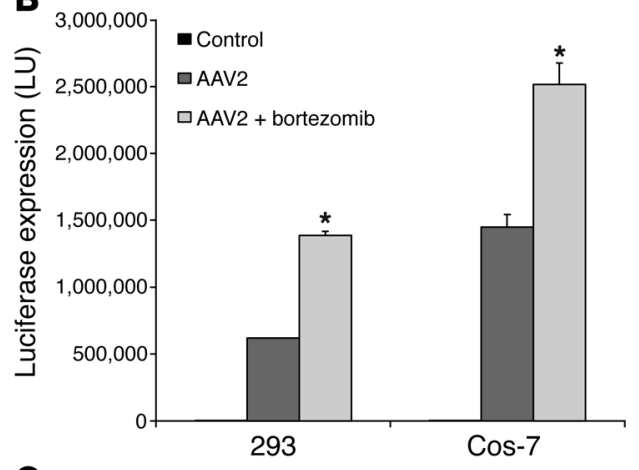

C

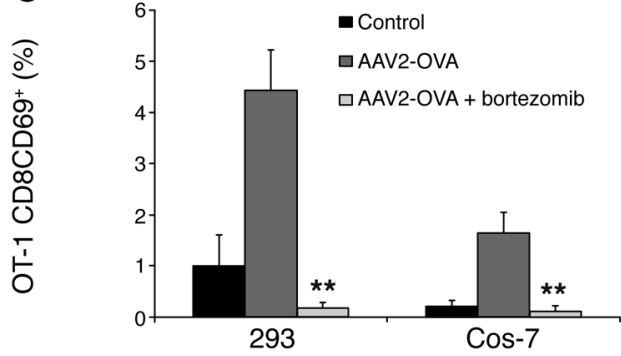

cell line (described above). We hypothesized that a proteasome-dependent degradation pathway may also apply to other AAV2-permissive cells from different origins. Two other cell lines - 293/ $\mathrm{H}-2 \mathrm{~Kb}$ and Cos- $7 / \mathrm{H}-2 \mathrm{~Kb}$ - were tested for this purpose. After 1-hour incubation of $293 / \mathrm{H}-2 \mathrm{~Kb}$ and Cos-7/H-2Kb cells with the proteasome inhibitor bortezomib, AAV2-OVA vectors were added to cell culture. Twenty-four hours later, the cells were fixed and cocultured with OT-1 spleen cells overnight, and thereafter CD8/CD69-expressing T cells were detected by flow cytometry. As shown in Figure 7, and consistent with results in the HepG2 cells, bortezomib completely inhibited antigen presentation by both AAV2-OVA-transduced 293 and Cos-7 cell lines (Supplemental Figure 6). Similar to prior studies, proteasome inhibitors enhanced AAV2 transduction in these 2 cell lines (Figure 7B).

\section{Discussion}

In this study, we demonstrate that capsid antigen presentation is dramatically inhibited by drugs that neutralize acidification of the endosome/lysosome, interfere with proteasome-mediated degradation, or block Golgi protein transport after AAV vector transduction in AAV-permissive cells. Furthermore, we found that AAV capsid antigen presentation was related to protein degradation by proteases and TPPII. Additional studies using well-characterized AAV capsid mutants have demonstrated that the inability of virions to escape from the endosome/lysosome resulted in decreased antigen presentation. Low antigen presentation was

\section{Figure 7}

Inhibition of capsid antigen cross-presentation with proteasome inhibitor in 293 and Cos7 cells. (A) Toxicity of bortezomib on 293/H-2Kb and Cos-7/H-2Kb cells. 293/H-2Kb cells $\left(2 \times 10^{5}\right.$ cells $)$ or Cos-7/H-2Kb cells $\left(2 \times 10^{5}\right.$ cells $)$ were seeded in 24 -well plates and incubated with bortezomib. Cell viability was measured 24 hours later. (B) Effect of bortezomib on AAV2 transduction in 293/H-2Kb cells and Cos-7/H-2Kb cells. 293 cells or Cos-7 cells were incubated with inhibitors for 1 hour, and then $1 \times 10^{4}$ particles per cell of AAV2/luc vector were added to the culture and cells were incubated for 24 hours, then harvested for luciferase analysis. ${ }^{*} P<0.05$ when compared with AAV2 without bortezomib treatment. (C) Effect of bortezomib on antigen presentation. After 293/H-2Kb cells or Cos-7/H-2Kb cells were incubated with bortezomib for 1 hour, they were treated with $2 \times 10^{10}$ AAV2-OVA/AAT vector for 24 hours, then fixed with $1 \%$ paraformaldehyde and washed. Next, OT-1 spleen cells were added to HepG2/H-2Kb cell culture medium. Activation of OT-1 spleen cells was determined by flow cytometry for detection of CD8 and CD69 expression (C). ${ }^{* *} P<0.01$ when compared with AAV2-OVA without bortezomib treatment. Data represent the average of 3 individual experiments and SD.

also observed using intact empty particles, whereas differential antigen presentation was observed when NLS/BR mutants (BR1, BR2, and BR3) were utilized.

The results of this study clearly show that the AAV capsid is processed by AAV2-permissive cells through an endosomal/cytosolic pathway (11). Exogenous antigen can be processed and presented via 2 main pathways: endosomal and cytosolic. Endocytosis is the primary approach used by AAV to transduce target cells. If the AAV2 virion can cross the endosomal/lysosomal barrier, antigen cytosolic processing will follow. If not, antigen processing will be completed within endosomes or lysosomes, and the peptides generated from protease degradation will bind to recycled MHC class I molecules to be presented on the cell surface. In light of this observation, a previous study showed that AAV particles can be found in endosomal vesicles shortly after AAV infection, and the lysosomotropic agents ammonium chloride and bafilomycin A1 prevent AAV-mediated transduction (26). In the present study, we demonstrate that the acidification inhibitors ammonium chloride and chloroquine blocked AAV capsid antigen presentation, suggesting that vacuolar acidification is involved in AAV capsid antigen processing. However, additional studies have determined that proteasomal transport via the Golgi complex is required to process $A A V$ particles and involves steps belonging to the cytosolic processing pathway (Figure 3 ). The data strongly suggest that capsid antigen presentation by AAV-transduced hepatocytes is proteasome dependent, given the need for acidification to allow $\mathrm{AAV}$ particle entry into the cytosol from the endosome/lysosome and for complete inhibition of AAV capsid antigen presentation by blocking proteasomal degradation. This conclusion is also in agreement with the fact that AAV antigen presentation is inhibited in the presence of BFA, which impedes protein transport (including nascent MHC class I molecules) from the ER, without affecting the recycling of MHC class I molecules in endosomes and lysosomes. These data also indicate that AAV2 capsid antigen is presented by newly synthesized MHC class I molecules.

Although AAV2 capsid antigen presentation is not endosome dependent via recycling MHC class I molecules, partial inhibition of AAV2 capsid processing and presentation was observed with the protease inhibitors pepstatin $\mathrm{H}$ and leupeptin $\mathrm{A}$, suggesting that proteolytic degradation in the endosome-lysosome system could 
be important in the initial steps of AAV2 virion degradation. It is interesting to speculate that proteolytic degradation allows AAV2 VP1 exposure on the virion surface, which in turn helps virions escape from the endosome/lysosome. It should not be overlooked that proteolytic degradation of the AAV capsid mediated by proteases could also occur in the autophagosome. Autophagy is a fundamental catabolic process that allows cells to recycle nutrients and maintain the quality and quantity of cellular cytoplasmic components (47). This process of self-digestion is essential for homeostasis, development, and differentiation in eukaryotic cells. During autophagy, a double-membrane vesicle, the autophagosome, is formed, which sequesters organelles, proteins, or parts of the cytoplasm, and fuses with the lysosome to degrade these intracellular components. Recently, autophagy has been proposed as another possible mechanism for antigen cross-presentation, especially for tumor antigen vaccine studies $(48,49)$, and it has been suggested that autophagy enhances classical MHC class I antigen presentation both in vitro and in vivo. In addition to its participation in antigen presentation of endogenous proteins by MHC class I molecules, autophagy may also be involved in antigen cross-presentation (49-51). After AAV infection, it is possible that AAV virions localized within organelles (e.g., Golgi, ER, microtubule organizing center [MTOC]) are sequestered by autophagosomes and degraded by proteases. The degraded AAV capsid peptides could then be delivered to the proteasome for further trimming to generate CTL epitopes, which bind to MHC class I molecules in the ER and present on the cell surface for CTL recognition. Continuing refinement of the role of autophagy in normal cell homeostasis should facilitate a better understanding of how these cellular processes contribute to virus-host interaction.

In the current paradigm, AAV2 transduction is initiated by capsid binding to HSPG as a primary receptor, followed by interaction with coreceptors (19-24). After binding, AAV2 is postulated to enter the target cells via receptor-mediated endocytosis into the acidic compartments in a clathrin- and dynamin-dependent process and travel to a perinuclear vesicle $(25,26)$. Acidification of the endosomal/lysosomal compartments is critical for effective AAV2 infection, and various domains within AAV capsids contribute to differential roles in endosomal escape and nuclear transport. For example, the catalytic activity of a PLA domain on the VP1 N-terminus is required for AAV2 infection. This domain is normally buried inside the AAV2 virion (27), however, during AAV2 infection, the PLA domain becomes exposed and its catalytic activity can now participate in endosomal release (27). Mutation of the PLA domain results in the loss of AAV2 infectivity, with defective trafficking of the AAV2 virion into the nucleus $(27,30)$. In this study, we also demonstrate that mutation of the PLA domain reduced AAV2 capsid antigen presentation, further supporting our pharmacological results which showed that the endosomal pathway is not the major mechanism for AAV capsid antigen cross-presentation after transduction.

In addition to the PLA domain, 3 NLS motifs (termed BR1, BR2, and BR3) with hydrophilic basic amino acid sequences in AAV2 capsid protein have been shown to play a critical role in AAV trafficking (29-31). Mutations in these capsid regions decrease AAV transduction. In this study, we examined the antigen presentation capacity of these mutants. Surprisingly, BR1 mutants generated less antigen presentation than wild-type AAV2, however, similar efficiency of antigen presentation was found among BR2 and BR3 mutants (defective for nuclear entry) and wild-type AAV2. This finding is different from that of the transduction efficiency of these mutants, in which BR1 mutants initiated higher transgene expression (4-fold lower than wild-type AAV2) than either the BR2 or BR3 mutants (100- and 2,000-fold lower than wild-type AAV2, respectively) (Figure 5). While the mechanism for these opposing phenomena is unclear, it is possible that the BR1 region mutant interferes with AAV2 capsid ubiquitination and decreases capsid degradation mediated by the proteasome. Alternatively, BR1 mutants de-route and target the Golgi complex, avoiding AAV2 capsid degradation (30). On the other hand, BR2 and BR3 mutants display a diffuse localization within the cytosol and may be more easily accessed by the ubiquitin-proteasome system (30). Since both BR2 and BR3 mutants are defective in nuclear transport, it can be concluded that nuclear entry by the AAV virion and uncoating in the nucleus are not necessary for effective capsid antigen cross-presentation. Whether antigens from uncoated capsids in the nucleus can be properly processed and presented warrants further investigation. Even though antigen presentation by capsids uncoated in the nucleus occurs, the efficiency is likely low compared with that of antigen from cytosol. Additionally, considering the high antigen presentation to low transgenic expression in BR2 and BR3 mutants, it seems that the AAV genome derived from virion uncoating in the cytoplasm is not permissive to entry into the nucleus and the subsequent induction of transgenic expression. These results further indicate that AAV virion uncoating occurs in both the nucleus and the cytoplasm.

During AAV production, a critical concern for clinical trials is the contamination of vector particles lacking a genome, especially when using purification approaches with column chromatography $(45,46)$. These empty virions substantially increase the dose of AAV capsid proteins and possibly result in an unwanted immunological response and decreased transgenic expression. Although empty AAV2 virions contain all components required for trafficking as full particles, including PLA2 and NLSs, capsid antigen presentation was nonetheless reduced in $\mathrm{HepG} 2 / \mathrm{H}-2 \mathrm{~Kb}$ cells infected with AAV2-empty virions compared with that in full particles. Although the fate of empty capsids in infected cells is not clearly known, the phenomenon of defective antigen presentation by AAV2-empty viruses may be interpreted as insufficient escape of these empty virions from the endosome. This interpretation is based on our previous study showing that AAV2-empty particles do not efficiently travel to the nucleus and are deficient in VP1 exposure (30). A recent study using cryoelectron microscopy and image reconstruction demonstrated that during packaging of the AAV genome, the AAV capsid experiences continuous conformational changes (52). It is possible that the conformational changes mediated by encapsidation of the AAV genome in full particles have a significant impact on exposure of the PLA domain on target cells during AAV infection. Three possibilities may account for the lack of effect of wild-type full particles from AAV2 virions on increasing antigen presentation by empty capsids. First, as described above, empty particles are unable to escape from endosomes. Second, there could be competition among the AAV transduction pathways, including receptor-binding competition between full particles and empty capsids, as was seen through inhibition of antigen presentation by AAV2-OVA full particles when coincubated with wild-type AAV2. A third option could be defective uncoating of empty AAV capsids in the cytosol. If empty virions are still intact in the cytosol, then the inability to present antigen would be consistent with published studies showing that intact virions are 


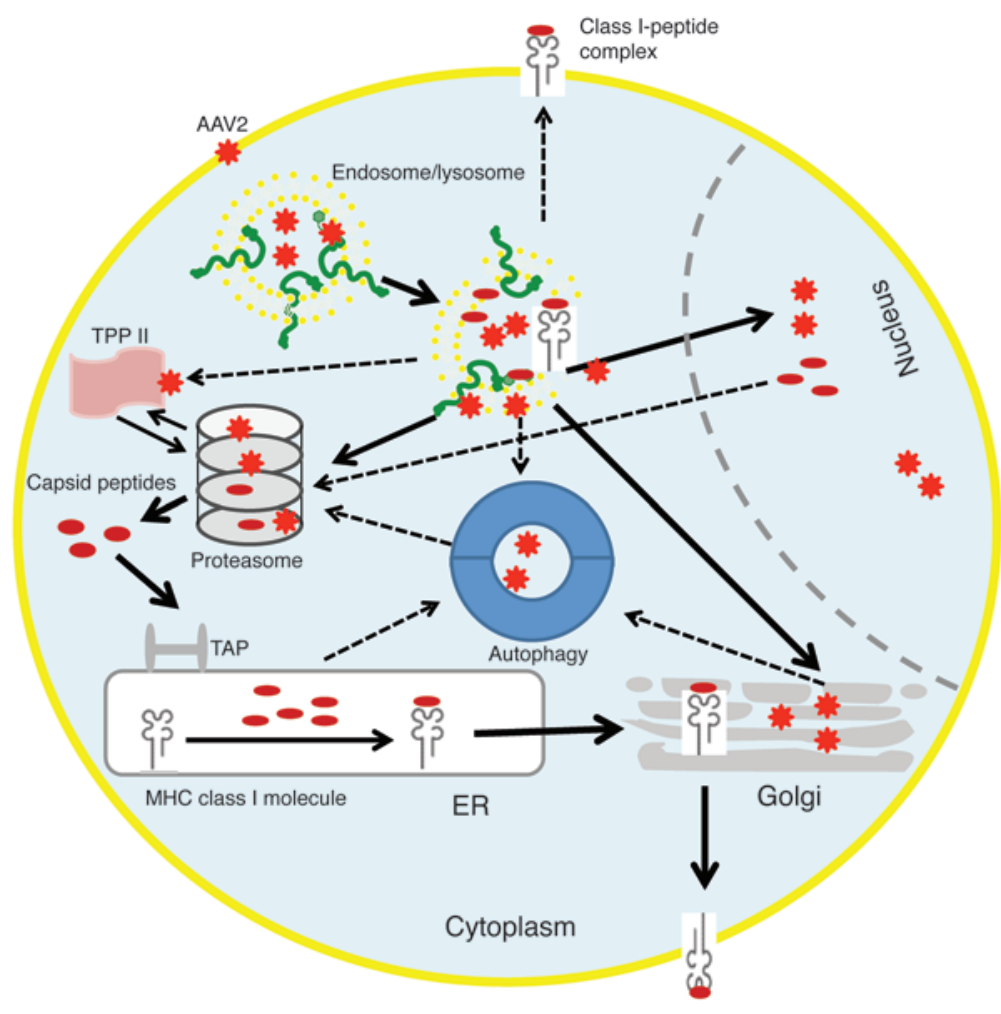

\section{Figure 8}

Putative model of AAV2 capsid antigen cross-presentation in AAV-transduced cells. Following binding on the cell surface in permissive cells via receptors and coreceptors, an AAV2 virion is uptaken by endocytosis into endosomes and lysosomes. PLA2 and NLSs are exposed on the virion surface to help the AAV virion escape into the cytosol. This is followed by AAV2 capsid ubiquitination and degradation by the proteasome. Resulting peptides are transported to the ER by TAP to bind $\mathrm{MHC}$ class I molecules. This complex is then trafficked to the cell surface via the Golgi network for recognition by capsid-specific CTLs. AAV2 virions also may travel to the Golgi complex and MTOC after endosomal/ lysosomal escape. These organelles are then sequestered by the autophagosome and delivered to proteasomes for capsid antigen processing, or AAV2 virions are released from the Golgi complex and the MTOC into the cytoplasm for proteasomal degradation. Additionally, uncoated AAV2 capsid may enter the proteasome after the AAV2 virion enters the nucleus. Solid line indicates the pathways from the prior studies and this study. Dotted line suggests possible routes for AAV2 capsid antigen processing and presentation. resistant to ubiquitination. If this is true, it suggests that exposure of the VP1 N-terminus is necessary for effective AAV uncoating and capsid degradation, consistent with published studies of AAV trafficking and LPA mutants. Since our experiments were performed in vitro over a relatively short period (24-48 hours) with respect to in vivo transduction (weeks), these studies may not reflect all the alternative capsid antigen processes that can take place over extended periods (e.g., empty capsids may be processed and presented over time after AAV transduction due to consistent interaction of these virions with protease in endosomes and/or cytosol). It should be noted that our finding of decreased antigen presentation by empty virions is inconsistent with a prior observation in which AAV2-empty pulsed target cells were killed by AAV capsid-amplified PBMCs (53). In the prior study, heterogeneous cell populations (NK, T cells) in PBMCs were used, and there was no negative control to determine whether cells without AAV2 infection were killed by AAV2-amplified PBMCs. Therefore, it is difficult to rule out the possibility of NK cell-mediated cytotoxicity. This possibility is supported by an antigen dose-response study in the same paper, where increased concentration of peptide resulted in higher CTL-mediated killing, but not for cells that were exposed to increased AAV vector doses (53).

The AAV virion is composed of 3 structural proteins: VP1, VP2, and VP3, with an approximate ratio of 1:1:8. It is unclear whether each virion contains the same ratio of 3 capsid proteins, or some virions contain only VP3. We show herein that antigen presentation by VP3-only virion is reduced compared with that by full, intact AAV2 particles, and this is consistent with the fact that VP3-only virions are deficient for endosomal escape due to a lack of PLA and NLSs (30).

In our previous study, we demonstrated that a strong cellular immune response could be elicited from AAV2 vector-pulsed DCs in mice, although no transgenic expression was observed in these cells (9). This finding provided obvious evidence that capsid antigen could be effectively processed and presented on the surface of APCs. It is unclear whether the intracellular pathways used by APCs for AAV capsid antigen processing and presentation are similar to those used by AAV2-permissive cells such as 293, Cos7, and HepG2.

In this study, we demonstrate that AAV2 capsid antigen cross-presentation is dependent on proteasome-mediated capsid degradation. This observation has very important clinical implications for the utilization of proteasome inhibitors: suppression of AAV capsid antigen presentation and enhancement of transgene expression from AAV transduction $(15,54)$. It has been demonstrated that proteasome inhibitors increase AAV transduction in vitro and in vivo $(15,38,54)$. The observation that bortezomib may influence AAV transduction as well as antigen presentation will be of significant importance when designing clinical studies using AAV vectors.

In a patient with hemophilia B, CTL-mediated elimination of transduced cells was observed at a later time point (4 weeks) after AAV delivery (1). Clinical studies have demonstrated that the elimination of transduced cells was not induced by transgene-specific (F9-specific) CTLs, but strongly suggested that AAV capsid-specific CTL response contributed to the therapeutic failure in this patient. We and others have suggested another possibility, which is that CTL response elicited from F9 cDNA alternative ORFs may be responsible for the clearance of AAV-transduced cells (33). CTL response detected in clinical trials was noted 4 weeks after infection, but results from this study show that antigen presentation by AAV2 capsid is dependent on the proteasome-mediated capsid degradation that occurs within the first 48 hours. It remains unknown why antigen from capsids in human hepatocytes are potentially presented very late after AAV transduction, and more importantly, it is unclear which pathway for this antigen cross-presentation is 
Table 1

Function and working concentration of inhibitors

Inhibitor
Chloroquine
Ammonium chloride
Leupeptin
Pepstatin
MG132
Bortezomib
Brefeldin A

AAF-CMK

\begin{tabular}{cc} 
Function & Working concentration \\
Prevents endosomal acidification & $100 \mu \mathrm{M}$ \\
Same as chloroquine & $20 \mathrm{mM}$ \\
Inhibits serine and cysteine proteases & $50 \mathrm{ng} / \mathrm{ml}$ \\
Inhibits aspartate proteases & $50 \mathrm{ng} / \mathrm{ml}$ \\
Blocks proteasomal activity & $10 \mu \mathrm{M}$ \\
Same as MG132 & $10 \mu \mathrm{M}$ \\
Prevents transport of ER resident MHC I & $5 \mu \mathrm{g} / \mathrm{ml}$ \\
molecules on the cell surface & \\
Inhibits TPPII & $500 \mathrm{ng} / \mathrm{ml}$ \\
\hline
\end{tabular}

Lentivirus vector $\mathrm{pTK} 642 / \mathrm{kb}$ was constructed by insertion of the blunted $\mathrm{H}-2 \mathrm{~Kb}$ fragment cut with EcoRI from PCBA-K2-kb into the HpaI site of pTK642. Endonuclease digestion and DNA sequencing were used to verify the clones.

Virus production. Recombinant AAV or lenti/ $\mathrm{H}-2 \mathrm{~Kb}$ was produced as described before in HEK293 cells (55). Briefly, for production of AAV, polyethylenimine (Sigma-Aldrich) was used for triple transfection of the pXR2 cap and rep plasmid, the pXX6-80 Ad helper plasmid, and the pTR-Luc or PTR-AAT reporter plasmids containing either luciferase or human alpha-1 antitrypsin transgenes flanked by inverted terminal repeats, respectively. Sixty hours later, 293 cells were harvested utilized. It is possible that both pathways (endosomal and proteasomal) of capsid antigen cross-presentation are involved in humans, however, the data derived from this in vitro study strongly suggest that further research using in vivo models may be required to elucidate this novel mechanism of capsid antigen cross-presentation after long-term AAV transduction in human liver.

According to results from AAV2 trafficking and data from this study, and as shown in Figure 8, we propose a putative model of AAV capsid antigen presentation by AAV-permissive cells. After cell surface binding and endocytosis, the AAV2 virion undergoes a conformational change in the endosome caused by an acidified environment. This process occurs in cooperation with the encapsidated AAV genome and/or proteases to expose PLA2 and NLS motifs on the virion surface for endosomal escape and trafficking to the nucleus. Virus that is ubiquitinated in the cytosol is delivered to proteasomes for degradation and generation of epitopes that bind to MHC class I molecules to form an MHC-I-peptide complex to present on the cell surface via the Golgi secretory pathway. TPPII is also involved in AAV capsid antigen processing either before or after AAV2 capsid degradation by the proteasome. Other organelles may be involved in AAV2 capsid antigen cross-presentation, thus after the AAV2 virion travels to the Golgi complex or the MTOC, an autophagosome may sequester these organelles and have them degraded by proteases. After further trimming of capsid peptides in the proteasome, the antigens are transported to the ER for loading onto MHC I molecules. Uncoated capsid in the nucleus may also be transferred to the proteasome for further degradation to generate peptides for antigen presentation.

In conclusion, AAV2 capsid antigen presentation requires endosomal escape and is proteasome dependent in AAV2-permissive cells. Also, encapsidation of the AAV genome is required, but uncoating in the nucleus is not necessary for effective cross-presentation. The results from this study will aid the research on effective approaches to avoid capsid-specific CTL-mediated elimination of AAV2-transduced target cells in future clinical trials.

\section{Methods}

Construction of recombinant plasmids. pXR2OVA, pXR2/AN75HD, and 4 BR mutant plasmids (pXR2-BR1OVA, pXR2-BR2, pXR2-BR3, and pXR2-VP3) were described in our previous studies $(29,30,33)$. To make $\mathrm{pXR} 2$-BR1OVA, pXR2-BR2OVA, pXR2-BR3OVA, pXR2-VP3OVA, and pXR2/AN75HDOVA, pXR2OVA was digested with $\mathrm{XcmI}$ and NotI to generate the fragment containing OVA SIINFEKL peptide nucleotide sequences. The fragment was cloned into pXR2-BR1, pXR2-BR2, pXR2-BR3, pXR2-VP3, and pXR2/ AN75HD-OVA by substituting the domain with $\mathrm{XcmI}$ and NotI digestion. and virus was purified by cesium chloride gradient density centrifugation. Virus was dialyzed against PBS and titered by dot-blot hybridization.

HIV-1-based vectors were generated using a transient 3-plasmid transfection approach with the standard calcium phosphate protocol (56). Briefly, vector constructs $(15 \mu \mathrm{g})$, vesicular stomatitis virus glycoprotein envelope expression cassette $(5 \mu \mathrm{g})$, and $\Delta \mathrm{NRF}$ packaging cassettes $(10 \mu \mathrm{g})$ were transfected into $293 \mathrm{~T}$ cells. Lentiviral particles were collected from conditioned medium 60 hours later. Lentivirus stocks were aliquoted and stored at $-80^{\circ} \mathrm{C}$. Cells. The hybridoma TIB139 was used to produce a monoclonal antibody against $\mathrm{H}-2 \mathrm{~Kb}$ (ATCC). HepG2/H-2Kb and 293/H-2Kb cell lines were established by transduction with lenti/ $\mathrm{H}-2 \mathrm{~Kb}$ in the presence of blasticidin $\mathrm{S}$ (InvivoGen). After transduction with lenti/H-2Kb, cells were incubated in medium with $10 \mu \mathrm{g} / \mathrm{ml}$ of blasticidin S. Blasticidin S-containing medium was replaced every 3-4 days. After 20 days of culture with blasticidin S, cells were serially diluted into 96-well plates at 1 cell per well and incubated in the presence of $50 \mu \mathrm{g} / \mathrm{ml}$ of blasticidin S. A single colony was transferred to a $10-\mathrm{cm}$ dish to establish the stable cell lines. H-2Kb expression on the cell lines was verified by flow cytometry. Cells were incubated with medium from TIB139 culture for 1 hour at $4^{\circ} \mathrm{C}$, and then washed. PE-conjugated rat antimouse Ig secondary antibody (BD Biosciences - Pharmingen) was added for 30 minutes at $4{ }^{\circ} \mathrm{C}$. Then the cells were washed and applied for cytometric analysis (Supplemental Figure 1). Cos7/H-2Kb cells were provided by $\mathrm{N}$. Shastri (University of California, Berkeley, California, USA). All cells were maintained at $37^{\circ} \mathrm{C}$ and $5 \% \mathrm{CO}_{2}$ in DMEM that was supplemented with $10 \%$ heat-inactivated FCS, $100 \mathrm{U} / \mathrm{ml}$ penicillin, and $100 \mathrm{~g} / \mathrm{ml}$ streptomycin.

Peptides. Peptides SIINFEKL (OVA 257-264) and p18 (F9 ARF1 18-26) (34) were synthesized at the Microprotein Sequencing and Peptide Synthesis Facility at the University of North Carolina, and were $90 \%$ pure. Peptides were reconstituted with DMSO at a concentration of $10 \mathrm{mg} / \mathrm{ml}$ and stored at $-20^{\circ} \mathrm{C}$.

Mice. The OT- 1 mouse is transgenic for an $\mathrm{H}-2 \mathrm{~Kb}$-restricted $\mathrm{T}$ cell receptor that recognizes the SIINFEKL peptide of OVA bound by $\mathrm{H}-2 \mathrm{~Kb}$ (Taconic Farms). All mice were maintained in a specific pathogen-free facility at the University of North Carolina at Chapel Hill. The University of North Carolina's IACUC approved all procedures for this study. Single spleen cells from OT-1 were obtained by grinding the spleen against a cell strainer. The red blood cells were lysed with lysis buffer (Sigma-Aldrich). After washing twice, the spleen cells were used for incubation experiments with AAV-transduced target cells.

Cell cytotoxicity assay. Cell viability with drug treatment was determined using the CellTiter-Glo Luminescent Cell Viability Assay kit (Promega). HepG2/H-2Kb cells $\left(2 \times 10^{5}\right)$ in $300 \mu$ lof culture medium were incubated with drugs at indicated concentrations in 12-well plates for 24 hours. Three hundred microliters of CellTiter-Glo Reagent (Promega) was added to each well and mixed for 2 minutes on an orbital shaker to induce cell 
lysis. The plate was allowed to incubate at room temperature for $10 \mathrm{~min}$ utes to stabilize luminescence signals. Luminescence was recorded with a Wallac 1420 Victor2 (PerkinElmer) automated plate reader.

Luciferase assay. HepG2/H-2Kb cells were plated in 24-well plates at a density of $10^{5}$ cells per well. Twenty-four hours after inoculation with $\mathrm{AAV}$ at $10^{4}$ particles per cell, the cells were lysed with passive lysis buffer (Promega). Luciferase activity was measured with a Wallac 1420 Victor2 (PerkinElmer) automated plate reader.

Antigen presentation assay. HepG2/H-2Kb, 293/H-2Kb, or Cos7/H-2b cells $\left(2 \times 10^{5}\right)$ were treated with inhibitors (Table 1$)$ for 1 hour and then infected with $2 \times 10^{10}$ particles of AAV2-OVA/AAT or AAV2/AAT virus for 24 hours at $37^{\circ} \mathrm{C}$, and $5 \% \mathrm{CO}_{2}$ in DMEM with $10 \%$ heat-inactivated FBS, $100 \mathrm{U} / \mathrm{ml}$ penicillin, and $100 \mathrm{~g} / \mathrm{ml}$ streptomycin. Then cells were fixed with $1 \%$ paraformaldehyde for 15 minutes at room temperature and washed 4 times using PBS with 3\% FBS. Fixed AAV-transduced cells were incubated with spleen cells from OT-1 mice for 24 hours. Cells were then harvested and washed, and PE-labeled rabbit anti-mouse CD69 antibody (BD Biosciences Pharmingen), along with an anti-CD8-FITC antibody (BD Biosciences Pharmingen), were added for 1 hour at $4^{\circ} \mathrm{C}$. OT- 1 spleen $\mathrm{T}$ cell activation was analyzed by flow cytometry for CD69 expression (an early marker for activated $\mathrm{T}$ cells). The efficiency of antigen presentation inhibition was calculated by:

[1 - (number of activated OT-1 cells in inhibitor group with AAV2OVA infection - background group without AAV2-OVA infection)/ (number of activated OT-1 cells in group with AAV2-OVA infection background group)] $\times 100 \%$.
The drug concentrations used in this study for all inhibitors were not toxic during a 24-hour culture period (Table 1 and Figure 3 ).

For antigen presentation analysis in $\mathrm{HepG} 2 / \mathrm{H}-2 \mathrm{~Kb}$ cells transduced with AAV2 mutants, VP3-only, or AAV2-empty virions, HepG2/H-2Kb cells were transduced with these viruses for 24 hours, then washed and incubated with OT-1 spleen cells for another 24 hours. T cell activation was determined by flow cytometry.

Statistics. A 2-tailed Student's $t$ test was used to compare means. A P value less than or equal to 0.05 was considered significant. All data were analyzed using Excel software (Microsoft).

\section{Acknowledgments}

We thank Xiaojing Chen and Karen Hogan for their excellent technical assistance. This work was supported by NIH grants 1R01AI080726 and 5R01DK084033 (to C. Li and R.J. Samulski), and 5U54AR056953 and 5R01AI072176 (to R.J. Samulski).

Received for publication September 5, 2012, and accepted in revised form December 5, 2012.

Address correspondence to: Chengwen Li or R. Jude Samulski, Gene Therapy Center, 7119 Thurston-Bowles, CB 7352, University of North Carolina at Chapel Hill, Chapel Hill, North Carolina 27599, USA. Phone: 919.962.3285; Fax: 919.966.0907; E-mail: chengwen@med.unc.edu (C. Li), rjs@med.unc.edu (R.J. Samulski).
1. Maguire AM, et al. Age-dependent effects of RPE65 gene therapy for Leber's congenital amaurosis: a phase 1 dose-escalation trial. Lancet. 2009; 374(9701):1597-1605.

2. Maguire AM, et al. Safety and efficacy of gene transfer for Leber's congenital amaurosis. $N$ Engl J Med. 2008;358(21):2240-2248.

3. Simonelli F, et al. Gene therapy for Leber's congenital amaurosis is safe and effective through 1.5 years after vector administration. Mol Ther. 2010; 18(3):643-650.

4. Ashtari M, et al. The human visual cortex responds to gene therapy-mediated recovery of retinal function. J Clin Invest. 2011;121(6):2160-2168.

5. Manno CS, et al. Successful transduction of liver in hemophilia by AAV-Factor IX and limitations imposed by the host immune response. Nat Med. 2006;12(3):342-347.

6. Nathwani AC, et al. Adenovirus-associated virus vector-mediated gene transfer in hemophilia B. NEngl J Med. 2011;365(25):2357-2365.

7. Chen J, Wu Q, Yang P, Hsu HC, Mountz JD. Determination of specific CD4 and CD8 T cell epitopes after AAV2- and AAV8-hF.IX gene therapy. $\mathrm{Mol}$ Ther. 2006;13(2):260-269.

8. Sabatino DE, et al. Identification of mouse AAV capsid-specific CD8 ${ }^{+} \mathrm{T}$ cell epitopes. Mol Ther. 2005; 12(6):1023-1033

9. Li C, et al. Adeno-associated virus type 2 (AAV2) capsid-specific cytotoxic T lymphocytes eliminate only vector-transduced cells coexpressing the AAV2 capsid in vivo. J Virol. 2007;81(14):7540-7547.

10. $\mathrm{Li} \mathrm{H}$, et al. Capsid-specific T-cell responses to natural infections with adeno-associated viruses in humans differ from those of nonhuman primates. Mol Ther. 2011;19(11):2021-2030.

11. Amigorena S, Savina A. Intracellular mechanisms of antigen cross presentation in dendritic cells. Curr Opin Immunol. 2010;22(1):109-117.

12. Burgdorf S, Scholz C, Kautz A, Tampe R, Kurts C. Spatial and mechanistic separation of cross-presentation and endogenous antigen presentation. Nat Immunol. 2008;9(5):558-566.

13. Gromme M, et al. Recycling MHC class I molecules and endosomal peptide loading. Proc Natl Acad Sci US A. 1999;96(18):10326-10331.

14. Ramachandra L, Simmons D, Harding CV. MHC molecules and microbial antigen processing in phagosomes. Curr Opin Immunol. 2009;21(1):98-104.

15. Finn JD, et al. Proteasome inhibitors decrease AAV2 capsid derived peptide epitope presentation on MHC class I following transduction. Mol Ther. 2010;18(1):135-142.

16. Ding W, Zhang L, Yan Z, Engelhardt JF. Intracellular trafficking of adeno-associated viral vectors. Gene Ther. 2005;12(11):873-880.

17. Ding W, Zhang LN, Yeaman C, Engelhardt JF. rAAV2 traffics through both the late and the recycling endosomes in a dose-dependent fashion. Mol Ther. 2006;13(4):671-682.

18. Nonnenmacher M, Weber T. Intracellular transport of recombinant adeno-associated virus vectors. Gene Ther. 2012;19(6):649-658.

19. Summerford C, Samulski RJ. Membrane-associated heparan sulfate proteoglycan is a receptor for adeno-associated virus type 2 virions. J Virol. 1998; 72(2):1438-1445

20. Qing K, Mah C, Hansen J, Zhou S, Dwarki V, Srivastava A. Human fibroblast growth factor receptor 1 is a co-receptor for infection by adeno-associated virus 2. Nat Med. 1999;5(1):71-77.

21. Summerford C, Bartlett JS, Samulski RJ. AlphaVbeta5 integrin: a co-receptor for adeno-associated virus type 2 infection. Nat Med. 1999;5(1):78-82.

22. Asokan A, Hamra JB, Govindasamy L, Agbandje-McKenna M, Samulski RJ. Adeno-associated virus type 2 contains an integrin alpha5beta1 binding domain essential for viral cell entry. J Virol. 2006; 80(18):8961-8969.

23. Akache B, Grimm D, Pandey K, Yant SR, Xu H, Kay MA. The 37/67-kilodalton laminin receptor is a receptor for adeno-associated virus serotypes 8, 2, 3, and 9. J Virol. 2006;80(19):9831-9836.

24. Kashiwakura Y, et al. Hepatocyte growth factor receptor is a coreceptor for adeno-associated virus type 2 infection. J Virol. 2005;79(1):609-614.

25. Duan D, Li Q, Kao AW, Yue Y, Pessin JE, Engelhardt JF. Dynamin is required for recombinant adeno- associated virus type 2 infection. J Virol. 1999; 73(12):10371-10376.

26. Bartlett JS, Wilcher R, Samulski RJ. Infectious entry pathway of adeno-associated virus and adeno-associated virus vectors. J Virol. 2000;74(6):2777-2785.

27. Girod A, et al. The VP1 capsid protein of adeno-associated virus type 2 is carrying a phospholipase A2 domain required for virus infectivity. J Gen Virol. 2002;83(pt 5):973-978.

28. Grieger JC, Johnson JS, Gurda-Whitaker B, Agbandje-McKenna M, Samulski RJ. Surface-exposed adeno-associated virus Vp1-NLS capsid fusion protein rescues infectivity of noninfectious wild-type $\mathrm{Vp} 2 /$ $\mathrm{Vp} 3$ and Vp3-only capsids but not that of fivefold pore mutant virions. JVirol. 2007;81(15):7833-7843.

29. Grieger JC, Snowdy S, Samulski RJ. Separate basic region motifs within the adeno-associated virus capsid proteins are essential for infectivity and assembly. J Virol. 2006;80(11):5199-5210.

30. Johnson JS, Li C, DiPrimio N, Weinberg MS, McCown TJ, Samulski RJ. Mutagenesis of adeno-associated virus type 2 capsid protein VP1 uncovers new roles for basic amino acids in trafficking and cell-specific transduction. J Virol. 2010; 84(17):8888-8902.

31. Sonntag F, Bleker S, Leuchs B, Fischer R, Kleinschmidt JA. Adeno-associated virus type 2 capsids with externalized VP1/VP2 trafficking domains are generated prior to passage through the cytoplasm and are maintained until uncoating occurs in the nucleus. J Virol. 2006; 80(22):11040-11054.

32. Pajusola K, Gruchala M, Joch H, Luscher TF, Yla-Herttuala S, Bueler H. Cell-type-specific characteristics modulate the transduction efficiency of adeno-associated virus type 2 and restrain infection of endothelial cells. J Virol. 2002;76(22):11530-11540.

33. Li C, et al. Cytotoxic-T-lymphocyte-mediated elimination of target cells transduced with engineered adeno-associated virus type 2 vector in vivo. J Virol. 2009;83(13):6817-6824.

34. Li C, et al. Cellular immune response to cryptic epitopes during therapeutic gene transfer. Proc Natl Acad Sci U S A. 2009;106(26):10770-10774. 
35. Umezawa H. Low-molecular-weight enzyme inhibitors of microbial origin. Annu Rev Microbiol. 1982; 36:75-99.

36. Mizuochi T, Yee ST, Kasai M, Kakiuchi T, Muno D, Kominami E. Both cathepsin B and cathepsin D are necessary for processing of ovalbumin as well as for degradation of class II MHC invariant chain. Immunol Lett. 1994;43(3):189-193.

37. Akache B, et al. A two-hybrid screen identifies cathepsins $\mathrm{B}$ and $\mathrm{L}$ as uncoating factors for adeno-associated virus 2 and 8. Mol Ther. 2007;15(2):330-339.

38. Johnson JS, Samulski RJ. Enhancement of adeno-associated virus infection by mobilizing capsids into and out of the nucleolus. J Virol. 2009; 83(6):2632-2644.

39. Tomkinson B, Lindas AC. Tripeptidyl-peptidase II: a multi-purpose peptidase. Int J Biochem Cell Biol. 2005; 37(10):1933-1937.

40. Seifert U, et al. An essential role for tripeptidyl peptidase in the generation of an MHC class I epitope. Nat Immunol. 2003;4(4):375-379.

41. Doms RW, Russ G, Yewdell JW. Brefeldin A redistributes resident and itinerant Golgi proteins to the endoplasmic reticulum. J Cell Biol. 1989; 109(1):61-72.

42. Pelham HR. Multiple targets for brefeldin A. Cell. 1991;67(3):449-451.
43. Gao G, et al. Purification of recombinant adeno-associated virus vectors by column chromatography and its performance in vivo. Hum Gene Ther. 2000; 11(15):2079-2091.

44. Lock M, Alvira MR, Wilson JM. Analysis of particle content of recombinant adeno-associated virus serotype 8 vectors by ion-exchange chromatography. Hum Gene Ther Methods. 2012;23(1):56-64.

45. Qu G, et al. Separation of adeno-associated virus type 2 empty particles from genome containing vectors by anion-exchange column chromatography. J Virol Methods. 2007;140(1-2):183-192.

46. Urabe M, et al. Removal of empty capsids from type 1 adeno-associated virus vector stocks by anionexchange chromatography potentiates transgene expression. Mol Ther. 2006;13(4):823-828.

47. Klionsky DJ, Emr SD. Autophagy as a regulated pathway of cellular degradation. Science. 2000; 290(5497):1717-1721.

48. Crotzer VL, Blum JS. Autophagy and its role in MHC-mediated antigen presentation. J Immunol. 2009;182(6):3335-3341.

49. Chemali M, Radtke K, Desjardins M, English L. Alternative pathways for MHC class I presentation: a new function for autophagy. Cell Mol Life Sci. 2011;68(9):1533-1541.

50. Uhl M, Kepp O, Jusforgues-Saklani H, Vicencio
JM, Kroemer G, Albert ML. Autophagy within the antigen donor cell facilitates efficient antigen cross-priming of virus-specific $\mathrm{CD}^{+} \mathrm{T}$ cells. Cell Death Differ. 2009;16(7):991-1005.

51. Li Y, Wang LX, Yang G, Hao F, Urba WJ, Hu HM. Efficient cross-presentation depends on autophagy in tumor cells. Cancer Res. 2008;68(17):6889-6895.

52. Gerlach B, Kleinschmidt JA, Bottcher B. Conformational changes in adeno-associated virus type 1 induced by genome packaging. J Mol Biol. 2011; 409(3):427-438.

53. Pien GC, et al. Capsid antigen presentation flags human hepatocytes for destruction after transduction by adeno-associated viral vectors. J Clin Invest. 2009;119(6):1688-1695.

54. Monahan PE, et al. Proteasome inhibitors enhance gene delivery by AAV virus vectors expressing large genomes in hemophilia mouse and dog models: a strategy for broad clinical application. Mol Ther. 2010;18(11):1907-1916

55. Xiao X, Li J, Samulski RJ. Production of hightiter recombinant adeno-associated virus vectors in the absence of helper adenovirus. J Virol. 1998; 72(3):2224-2232.

56. Naldini $\mathrm{L}$, et al. In vivo gene delivery and stable transduction of nondividing cells by a lentiviral vector. Science. 1996;272(5259):263-267. 